\title{
SIMULAÇÃO NUMÉRICA DE FLUXOS DE CALOR RECEBIDOS EM VESTUÁRIO DE PROTEÇÃO DE BOMBEIRO
}

\section{RESUMO}

\author{
Salim Dahamni ${ }^{1}$ \\ Khedouma Nechab ${ }^{2}$ \\ Abdallah Benarous ${ }^{3}$ \\ Paulo A. G. Piloto ${ }^{4}$
}

Neste estudo é apresentada uma simulação numérica para determinar o fluxo térmico recebido pelo vestuário de proteção individual utilizado pelos bombeiros. O estudo é centrado no impacto das condições de ventilação dos compartimentos nos fluxos incidentes que atingem a superfície exterior do equipamento de proteção individual (EPI). É apresentada uma simulação de transferência de calor por radiação e por convecção para um compartimento com geometria definida a três dimensões, constituído por uma porta, uma janela e sujeito a uma fonte de calor localizada, caracterizado por uma taxa estacionária de liberação de calor (HRR) durante um tempo de exposição prescrito. É considerada uma restrição aditiva no limiar da fração mássica de oxigênio responsável pela extinção da chama. A camada exterior do vestuário de proteção é modelada como um meio sólido, com sensores posicionadas no peito ( $F$ - front) e atrás do peito (R-rear). $O$ caso apresentado corresponde a uma situação em que a temperatura exterior do vestuário proteção e a do ar fresco adjacente ao corpo do bombeiro são mantidas a $25^{\circ} \mathrm{C}$. Apesar da importância dos dispositivos de ventilação mecânica para eliminação do fumo e atenuação da temperatura, são determinados valores críticos para os caudais de ventilação que podem levar a enormes fluxos de calor, revelando a aparição de situações de ignição instantânea de combustão incompleta (FOV).

Palavras-chave: Vestuário de bombeiro; Fluxo de calor; Ventilação mecânica; Incêndio generalizado.

\footnotetext{
${ }^{1}$ Lecturer. Université Hassiba Benbouali de Chlef. Algerie

${ }^{2}$ PhD student, Université Hassiba Benbouali de Chlef. Algerie

${ }^{3}$ Associate Professor. Université Saad Dahlab de Blida 1. Blida. Algerie

${ }^{4}$ Coordinator Professor. Instituto Politécnico de Bragança. Portugal
} 


\title{
NUMERICAL SIMULATION OF INCOMING THERMAL FLUXES ON A FIREFIGHTER GARMENT
}

\begin{abstract}
The present study is a numerical attempt for the prediction of the incoming thermal flux on a firefighter protective clothing. The study focuses on ventilation conditions impact on the incident fluxes reaching the external garment's face. A radiative and convective transfer modelling is considered for a $3 \mathrm{D}$ geometry compartment, equipped with a door, a window and subject to a localized fire, with stationary heat release rate (HRR) during a prescribed exposure time. An additive constraint on oxygen mass fraction threshold is considered to account for flame extinction. The outer layer of the protective garment is modelled as a solid medium, featuring both front (chest F) and rear (back $R$ ) sensors. The baseline case corresponds to a situation where the external temperature of the protective clothing and that of the fresh air adjacent to the firefighter's body are maintained at $25^{\circ} \mathrm{C}$. Despite the importance of mechanical ventilation devices in smoke clearance and temperature attenuation, critical values for ventilation flow rates may lead to tremendous heat fluxes revealing the existence of backdraft situations.
\end{abstract}

\section{INTRODUÇÃO}

O fogo é um dos acidentes mais destrutivos que ameaçam a vida das pessoas e a durabilidade das estruturas. Os bombeiros ou ocupantes podem ficar submetidos a um ambiente de radiação e convecção térmica, incluindo ambientes químicos perigosos, gases tóxicos e fluxos de calor significativos 
causados pela combustão de materiais ou pelo transporte de fumo (Lassus et al, 2016). Os bombeiros devem usar vestuário de proteção individual que ofereçam proteção eficaz contra vários tipos de exposições térmicas, como chamas e fumo. A utilização frequente de material de isolamento sintético contribui para o aumento da carga térmica, podendo exibir um comportamento inesperado durante um combate a incêndios ( $\mathrm{He}$ et al. 2015). Durante a ocorrência de um incêndio, os bombeiros são submetidos a níveis variáveis de fluxos de calor. Apesar de se poderem encontrar submetidos a valores de baixa densidade de fluxo de calor (Baczkiewicz et al. 2018), a permanência a longos períodos de exposição pode proporcionar um desequilíbrio térmico do corpo humano e queimaduras na pele (Fu et al. 2015).

$O$ incêndio no compartimento é conduzido por fenómenos complexos que foram objeto de muitas investigações. A ventilação natural (NV) é um dos tópicos que atrai os investigadores a desenvolver ensaios experimentais e estudos de dinâmica de fluidos computacional (CFD) para modelos reduzidos e para modelos à escala real (Mvogo et al. 2019). Neste sentido, foram desenvolvidos ensaios experimentais para estudar o efeito de abertura horizontal no comportamento do fogo de líquido combustível (Chen et al. 2016). Em tais situações, a razão de equivalência do compartimento mostrou-se fortemente relacionada com os modos de autoextinção deste tipo de incêndio.

Os estudos sobre os incêndios generalizados têm sido geralmente limitados a cálculos do risco de incêndio, nos quais são presumidas curvas da taxa de liberação de calor (HRR) (Babrausskas et al. 2003). No entanto, existem poucos relatos de estudos sobre o incêndio generalizado causado por uma re-ignição natural dos gases inflamáveis (Zang et al. 2014). Portanto, é importante destacar a contribuição da ventilação natural e da ventilação mecânica para o desenvolvimento de fluxos térmicos em corpos sólidos específicos durante um determinado tempo de exposição.

$O$ presente trabalho tem como objetivo simular numericamente os fluxos convectivos e radiativos recebidos pelo vestuário, como equipamento individual 
de proteção. Foi considerada a libertação de calor de uma fonte de combustível líquido, dentro de um compartimento confinado, ventilado naturalmente (NV) e ventilado mecanicamente (MV). Situações específicas relacionadas com a subventilação e com a re-ignição dos materiais combustíveis são apresentadas e discutidas em relação à intensidade dos fluxos de calor recebidos pelas superfícies do vestuário do bombeiro.

\section{MODELO DE TRANSFERÊNCIA DE CALOR}

Em situação de incêndio, os fluxos de calor geralmente têm origem nas superfícies mais quente, como chamas, plumas ou superfícies sólidas (paredes). A contribuição de parte de transferência de calor por radiação provou ser importante quando se verifica um determinado nível de confinamento no compartimento (Rossi et al. 2011). Além disso, fluxos convectivos significativos podem surgir em situações de ventilação natural ou mecânica, permanecendo relativamente baixos quando a chama estabiliza, mas intensificando-se em algumas situações críticas (Nayak et al. 2014).

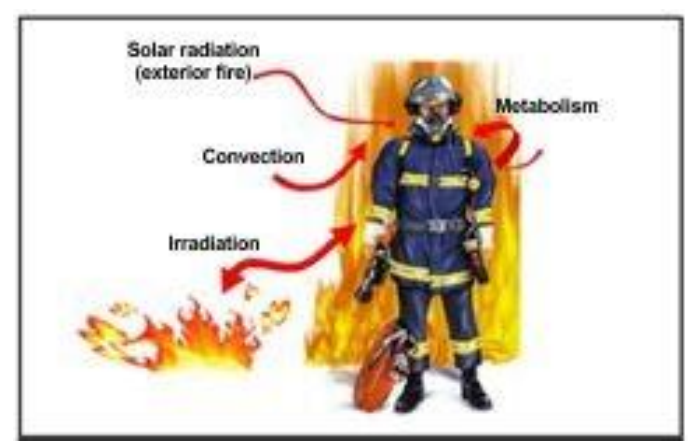

Figura 1: Fontes de calor para o equilibro térmico de um bombeiro (Douadi 2018).

A chama localizada que resulta de um incêndio de material combustível é a fonte primária que transmite o fluxo radiativo para qualquer superfície. Nos modelos de aproximação de engenharia, podem ser utilizados modelos simplificados para a previsão dos fluxos recebidos pela superfície (Nayak et al. 
2014). Para tais situações, a expressão correspondente do fluxo recebido é dada como:

$$
\phi_{\text {rad }}=F \times \tau \times \phi_{f}
$$

onde $\phi_{\text {rad }}$ representa o fluxo de calor recebido na superfície $\left(\mathrm{kW} / \mathrm{m}^{2}\right)$, F representa $\circ$ fator de forma, $\tau$ representa 0 fator de transmissividade atmosférica e $\phi_{f}$ define o fluxo de calor emitido pelo incêndio localizado de combustível, que se relaciona com a liberação de calor da chama (Qf). No modelo simplificado de chama permanente, a potência de emissão é considerada uniforme em toda a altura da chama $\mathrm{H}_{\mathrm{f}}$. Portanto, o fator de atenuação devido à absorção e difração por infravermelho pode ser expresso de acordo com a correlação de Brzustowski e Sommer (Douadi 2018):

$$
\tau=0.79 \times(100 / d)^{1 / 16} \times(30.5 / R H)^{1 / 16}
$$

d é a distância entre o corpo do bombeiro e a fonte de chama; $\mathrm{RH}$ é a humidade relativa do ar interno do compartimento, sendo geralmente igual a $70 \%$. O fator de forma depende da geometria considerada para a chama, pelo que quando a chama é vista como uma superfície em forma de paralelepípedo, este fator pode ser determinado através de (Douadi 2018):

$$
F_{v}=1 / 2 \pi \times\left[X / \sqrt{1+X^{2}} \times \operatorname{Arctg}\left(Y / \sqrt{1+X^{2}}\right)+Y / \sqrt{1+Y^{2}} \times \operatorname{Arctg}\left(X / \sqrt{1+X^{2}}\right)\right]
$$

com $X=H_{f} / d$ e $Y=D_{f} / d$, em que $D_{f}$ se refere ao diâmetro equivalente da chama. A altura média da chama é expressa através da correlação de Heskestad como (Douadi 2018):

$$
H_{f} / D_{f}=-1.02+0.235 \times Q_{f}^{2 / 5} / D_{f}
$$


A taxa de liberação de calor da chama $\left(Q_{\mathrm{f}}\right)$ está relacionada com o tipo de combustível e com a taxa de mistura ar-combustível. No entanto, para aplicações de engenharia, podem ser consideradas curvas padrão de taxa de liberação de calor, tendo em consideração as condições de ventilação (Corrêa et al 2018). De acordo com o conhecimento dos autores, não existem formulações analíticas que permitam fazer a previsão de fluxos convectivos recebidos, na presença de ventilação natural ou mecânica.

\section{DISCUSSÃO DE RESULTADOS}

O compartimento em estudo consiste em uma sala de $10 \mathrm{~m} \times 4 \mathrm{~m} \times 2,9 \mathrm{~m}$, com uma porta e uma janela. A área de combustível possui $1,5 \mathrm{~m}^{2}$ de superfície, está localizada nas proximidades da janela e é responsável pela taxa de liberação de calor estacionária $Q_{\mathfrak{f}}=1125$ W (Fig.2).

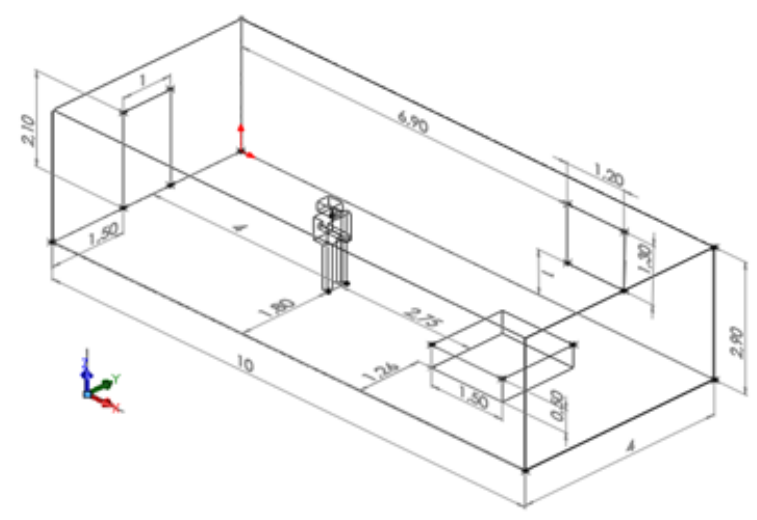

Figure 2: Perspetiva da geometria do compartimento (Douadi 2018).

O corpo do bombeiro é modelado como uma placa plana, localizada a uma distância padrão $(2,75 \mathrm{~m})$ do incêndio localizado, na qual são aplicados dois sensores de fluxo de calor, respetivamente, na frente $(F)$ e na parte traseira (R). A densidade de fluxo de calor emitida de pela chama $Q_{f}=500 \mathrm{~kW} / \mathrm{m}^{2}$ é 
estacionária durante o período de observação tphys $=15$ minutos (Desanghere 2006). As paredes e a face externa do corpo do bombeiro são mantidas a uma temperatura $\mathrm{T}=25{ }^{\circ} \mathrm{C}$ (Fig.3.a) e o domínio de computação é mapeado com uma malha estruturada contendo 100000 células (Fig.3.b). A técnica de simulação de escoamentos turbulentos (LES - Large Eddy Simulation) é usada para resolver as equações de Navier-Stokes, transferência de energia e radiação, utilizando o programa Fire Dynamic Simulator FDS ${ }^{\circledR}$.

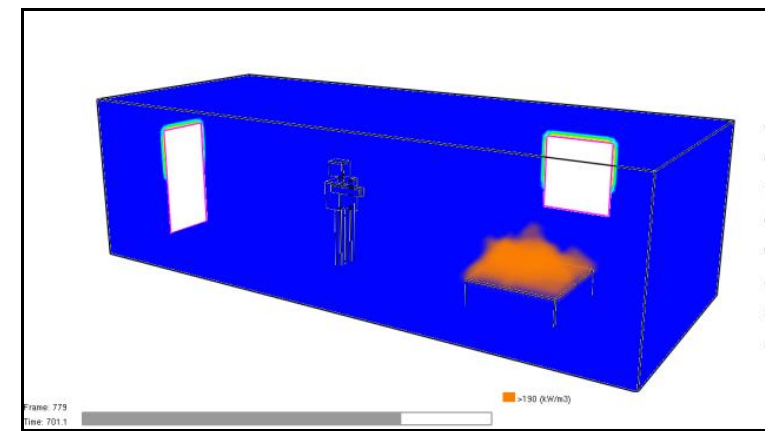

Figura 3.a: Condições fronteira (Dahamni 2016).

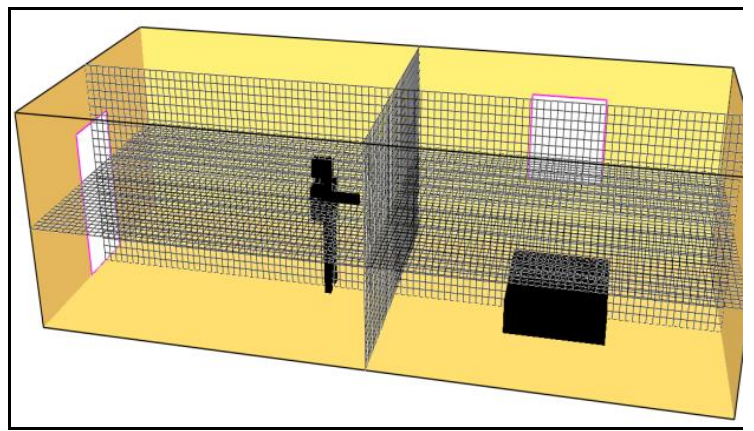

Figura 3.b: Malha estruturada para 0 domínio computacional.

Considerando a libertação de calor em regime estacionário na região do combustível, os fluxos de calor de convecção e radiação incidentes no corpo do bombeiro estão relacionados com os regimes de ventilação da sala e, mais especificamente, com as condições de regulação da entrada de ar na base da chama. Por conseguinte, foram estudadas três configurações possíveis para o local: confinamento total (porta fechada, janela fechada), ventilação natural (NV - uma ou duas aberturas livres) e ventilação mecânica (MV - insuflação de ar).

A Figura 4 mostra as curvas de evolução dos fluxos recebidos pelo corpo do bombeiro, em situação de confinamento total (fator de abertura OF = 0). Observa-se um aumento abrupto no fluxo de calor de convecção recebido através do corpo do bombeiro durante os primeiros 60 segundos, que revela uma resposta instantânea, com um fluxo máximo de $2,2 \mathrm{~kW} / \mathrm{m}^{2}$, recebido na superfície frontal e um fluxo convectivo de $2 \mathrm{~kW} / \mathrm{m}^{2}$, recebido na superfície traseira (fig.4.a). Esse aumento é explicado por uma variação significativa na 
densidade do ar entre o local da chama e o local do bombeiro $(\infty)$. No entanto, a partir dos $180 \mathrm{~s}$, o fluxo convectivo diminui até um valor zero (últimos $540 \mathrm{~s}$ ), provocado pela diminuição da intensidade da chama e à ausência de circulação de ar ao redor do bombeiro.

Em relação ao fluxo de calor recebido por radiação, foi considerado um valor inicial de $0,5 \mathrm{~kW} / \mathrm{m}^{2}$, registrado para o instante inicial, que corresponde ao valor da fonte de fogo (fig.4.b). O fluxo de calor por radiação recebido no tórax é mais importante, com uma resposta instantânea $q_{\text {rad,inc }}=4,5 \mathrm{~kW} / \mathrm{m}^{2}$, para o instante $5 \mathrm{~s}$, variando de $4,5 \mathrm{~kW} / \mathrm{m}^{2}$ até um valor máximo de $6,5 \mathrm{~kW} / \mathrm{m}^{2}$. No entanto, o fluxo de calor de radiação incidente na parte traseira permanece constante com um valor moderado $\left(5,5 \mathrm{~kW} / \mathrm{m}^{2}\right)$ em relação ao fluxo de calor recebido no tórax ( $\mathrm{q}_{\text {front }} \sim 1,1 \mathrm{q}_{\mathrm{rear}}$ ), devido ao efeito sombreado da superfície frontal do bombeiro. A partir do instante de tempo 180s, o fluxo de radiação recebido pelo corpo do bombeiro diminui assintoticamente em direção a um valor limite de $0,5 \mathrm{~kW} / \mathrm{m}^{2}$, que corresponde ao fluxo emitido pelos gases ao redor. De maneira geral, o fluxo de calor de radiação recebido pelo corpo do bombeiro é maior que o fluxo de calor de convecção $\left(q_{\text {rad }} \sim 3 q_{\text {con }}\right)$.

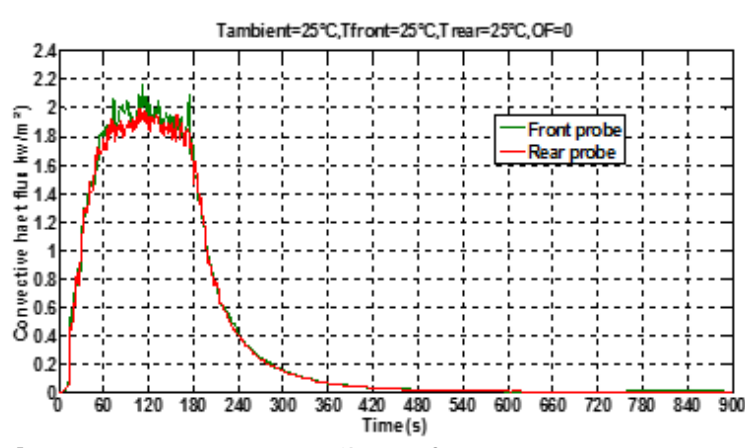

Figura 4.a: Evolução o fluxo de calor de convecção recebido pelo bombeiro.

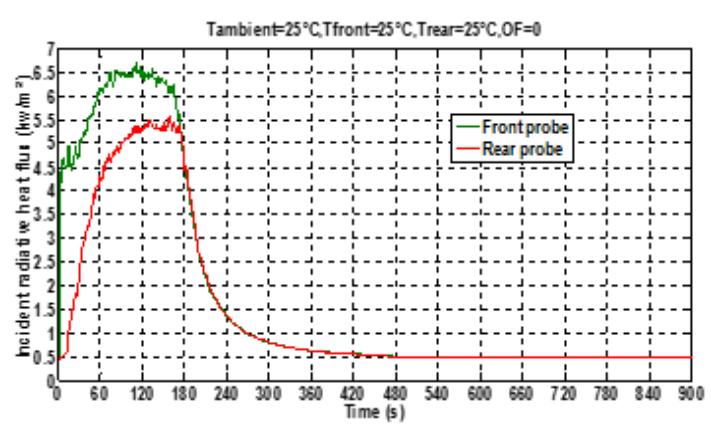

Figura 4.b: Evolução o fluxo de calor de radiação recebido pelo bombeiro.

Para o caso correspondente a uma ventilação natural, assumindo a porta e a janela abertas (OF $=0,045$ ), a amplitude dos fluxos parece um pouco atenuada. A Figura 5.a mostra a variação do fluxo de calor por convecção recebido pelo corpo do bombeiro, em função do tempo de exposição. 
A Figura 5.b representa a variação do fluxo de calor de radiação recebido pelo bombeiro. Este valor varia entre $4,5 \mathrm{~kW} / \mathrm{m}^{2}$ e $5 \mathrm{~kW} / \mathrm{m}^{2}$ na face frontal e $2 \mathrm{~kW} / \mathrm{m}^{2}$ a $2,5 \mathrm{~kW} / \mathrm{m}^{2}$ na parte traseira, com uma relação $\mathrm{q}_{\text {front }} \sim 2 \mathrm{q}_{\text {rear. }}$. Também se conclui que a amplitude do fluxo de calor recebido por convecção ainda é menor em comparação com o fluxo de radiação ( $\left.q_{r a d} \sim 4 q_{c o n}\right)$.

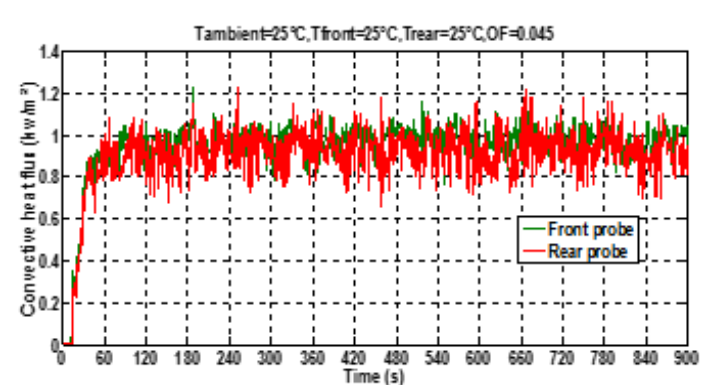

Figura 5.a: Evolução o fluxo de calor de convecção recebido pelo bombeiro.

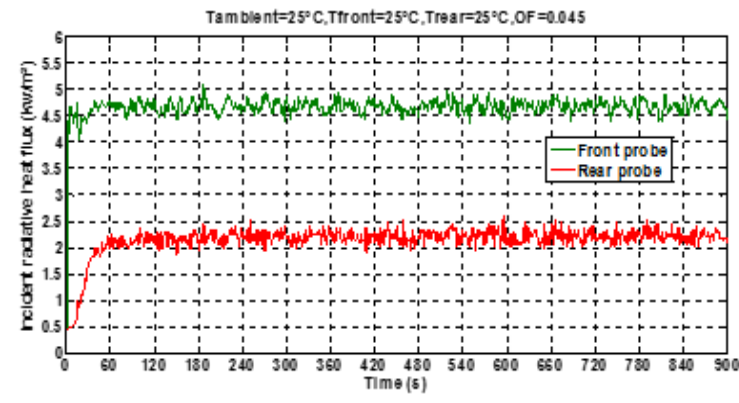

Figura 5.b: Evolução o fluxo de calor de radiação recebido pelo bombeiro.

Comparando estes resultados com os resultados da simulação de compartimento confinado, os fluxos parecem mais estáveis (sem extinção), variando de $0,7 \mathrm{~kW} / \mathrm{m}^{2}$ a $1,2 \mathrm{~kW} / \mathrm{m}^{2}$. Esses limites são menos intensos e revelam o efeito da evacuação natural do fumo gerado, através das aberturas (Fig.6).

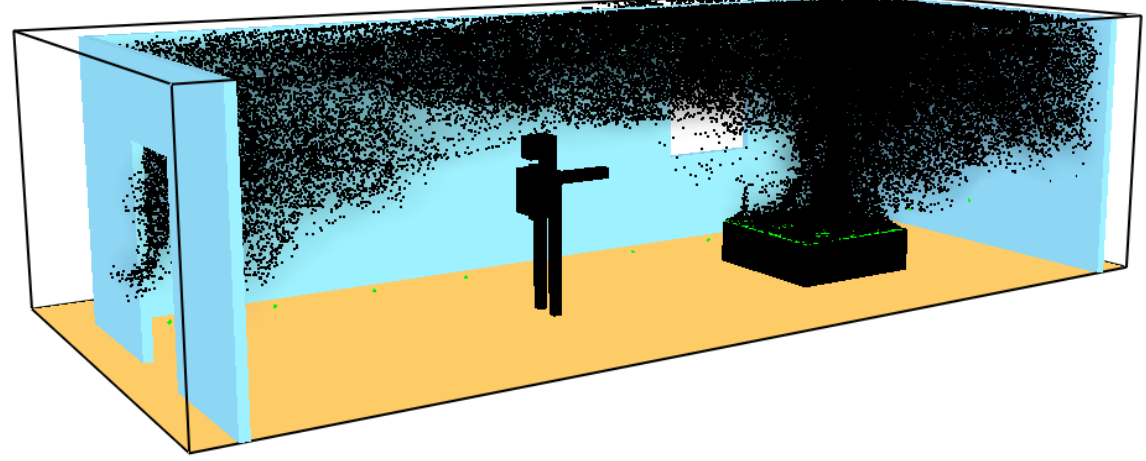

Figura 6: Evacuação do fumo pelas aberturas. 
A ventilação mecânica envolve a insuflação de ar novo através de uma ou mais aberturas para diminuir a temperatura e evacuar o fumo gerado. $O$ caso apresentado corresponde a uma configuração em que a porta e a janela estão abertas ( $O F=0,045)$ e a insuflação é gerada através da porta com um caudal de $0,54 \mathrm{~m}^{3} / \mathrm{s}$. A Figura 7.a mostra a variação do fluxo de calor de convecção recebido pelo corpo do bombeiro neste compartimento semiconfinado. Apresenta um ligeiro aumento em comparação com o caso de ventilação natural. No entanto, o fluxo convectivo recebido na face traseira do bombeiro mostra uma variação entre $1,3 \mathrm{~kW} / \mathrm{m}^{2}$ e $1,5 \mathrm{~kW} / \mathrm{m}^{2}$. Isto é principalmente devido ao fluxo de ar fornecido pelo sistema de ventilação da porta, localizado atrás do bombeiro.

A Figura 7.b mostra uma flutuação estável do fluxo de calor de radiação recebido pela superfície frontal do bombeiro, com valores a variar entre 4,5 $\mathrm{kW} / \mathrm{m}^{2}$ e $5 \mathrm{~kW} / \mathrm{m}^{2}$. Estes resultados são um pouco maiores que os do caso de ventilação natural. De facto, a ventilação da sala acelera a combustão, pois adiciona oxigénio, o que aumenta a quantidade do fluxo de calor de radiação emitido pela chama.

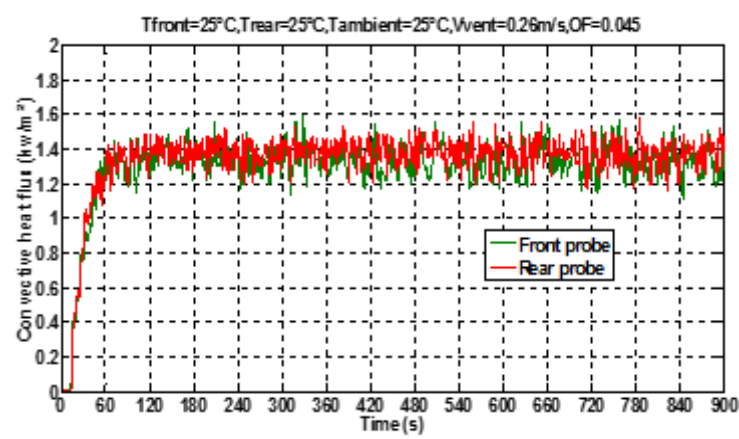

Figura 7.a: Evolução o fluxo de calor de convecção recebido pelo bombeiro.

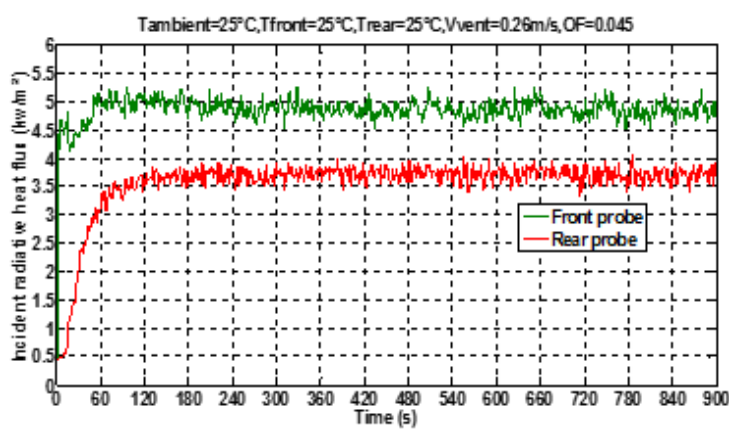

Figura 7.b: Evolução o fluxo de calor de radiação recebido pelo bombeiro.

O fluxo de calor recebido pela superfície traseira também aumentou. A influência do fluxo de radiação recebido na superfície traseira do bombeiro depende muito das condições de ventilação impostas pelo ar insuflado na porta. A quantidade de calor de radiação recebido varia entre $3,5 \mathrm{~kW} / \mathrm{m}^{2}$ e até 4 
$\mathrm{kW} / \mathrm{m}^{2}$, o que apresenta um aumento significativo em relação a um espaço semi-confinado com ventilação natural. $O$ aumento do valor pode ser explicado pelo fato de que uma quantidade do fluxo radiativo emitido pelo fumo é acumulada ao nível da superfície traseira do corpo do bombeiro.

Foi ainda simulada a condição em que a janela permanece fechada e se começa a insuflar ar novo pela porta (fator de abertura OF $=0,026$ ), assumindo um caudal crítico de $0,42 \mathrm{~m}^{3} / \mathrm{s}$, situação considerada muito frequente em relação às manobras de combate a incêndios. Nestas condições, os fluxos de entrada parecem temporariamente atenuados, situação justificada pela insuficiente ventilação do compartimento. Verifica-se que o calor recebido cai para valores nulos após o instante $180 \mathrm{~s}$, verificando-se um aumento instantâneo do calor recebido antes do final da simulação (15 min), induzindo a condição de incêndio generalizado (FOV) com libertação de fluxo de calor elevados, que excedem os limites de tenacidade do vestuário de proteção dos bombeiros (Fig.8.a, 8.b).

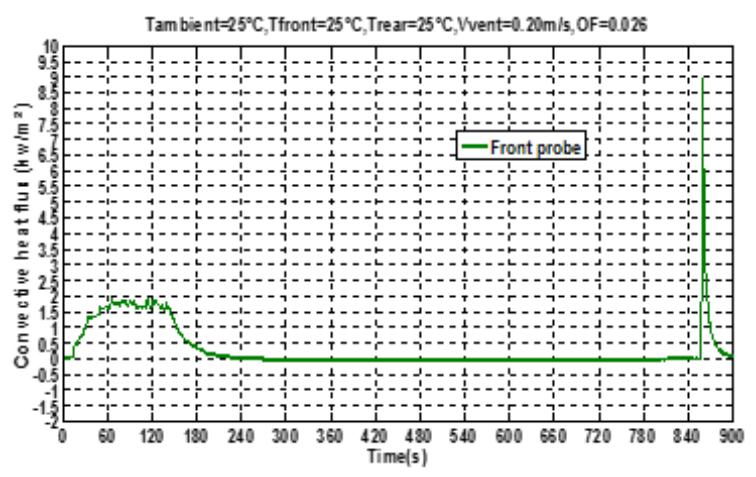

Figura 8.a: Evolução o fluxo de calor de convecção recebido pelo bombeiro (FOV).

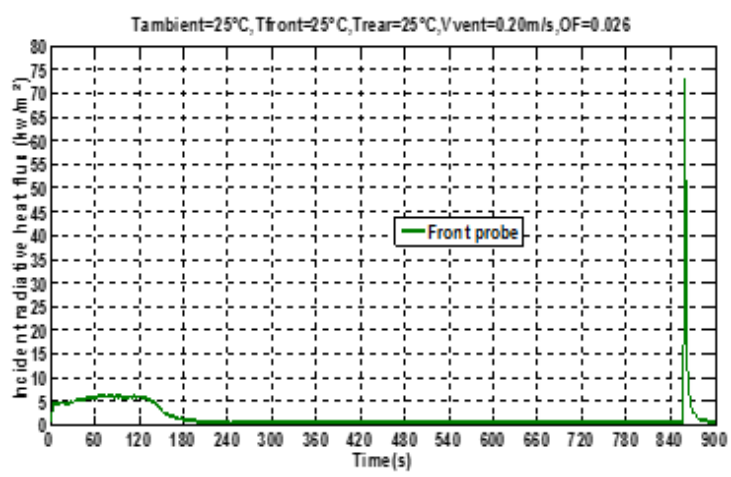

Figura 8.b: Evolução o fluxo de calor de radiação recebido pelo bombeiro (FOV).

A mesma situação é verificada quando se considera a insuflação de ar pela janela, assumindo um caudal crítico de $0,39 \mathrm{~m}^{3} / \mathrm{s}$, mantendo a porta fechada ( $O F=0,019)$. Nesta condição também se verifica um aumento instantâneo do calor recebido atingindo a condição de incêndio generalizado (FOV), imediatamente após o instante 16 minutos (Fig 9.a, 9.b). 

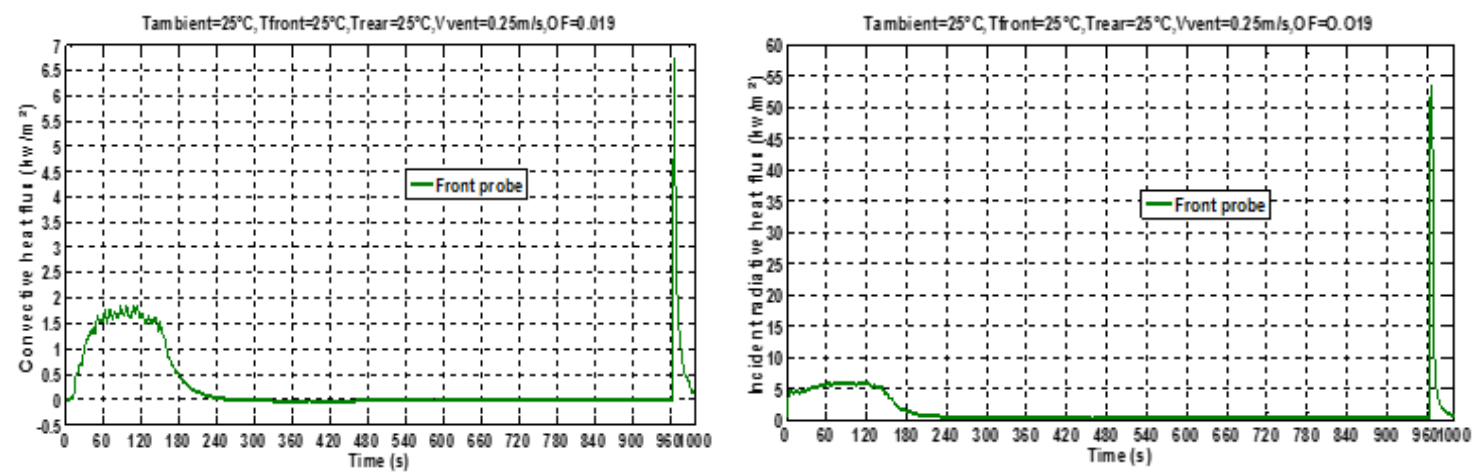

Figura 9.a: Evolução o fluxo de calor Figura 9.b: Evolução o fluxo de calor de de convecção recebido pelo bombeiro radiação recebido pelo bombeiro (FOV). (FOV).

Os limiares térmicos atingidos nesta situação são relativamente mais baixos que os do primeiro caso de incêndio generalizado, sendo justificado pelo valor inferior do caudal de insuflação de ar. Consequentemente, situações repentinas de incêndio generalizado afetam a temperatura nas proximidades do bombeiro (Fig. 10). Na Figura 10 estão representados três instantes de tempo representativos da variação de temperatura no compartimento.

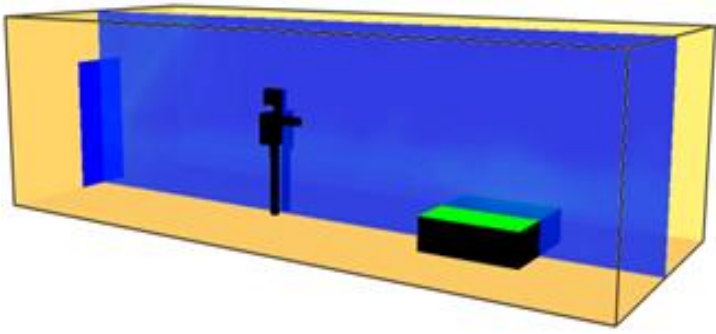

a) Instante $855 \mathrm{~s}$.

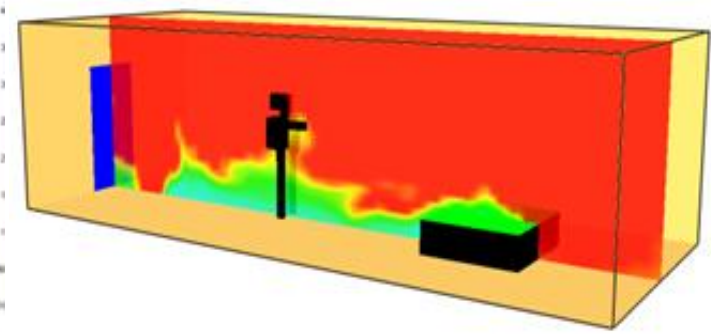

b) Instante $860 \mathrm{~s}$.

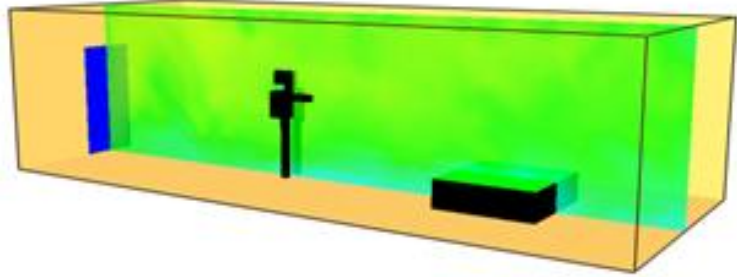

c) Instante $865 \mathrm{~s}$

Figura 10.a: Campo de temperatura no compartimento de incêndio durante a fase de incêndio generalizado (FOV), para a condição de compartimento ventilado $(\mathrm{OF}=0.026)$. 


\section{CONCLUSÕES}

O presente estudo representa um conjunto de simulações numéricas dos fluxos térmicos recebidos pelo equipamento de proteção individual externa do bombeiro (vestuário). É apresentado um modelo simplificado para o cálculo do fluxo de calor recebido por uma superfície do EPI de um bombeiro, provocado por um incêndio localizado num compartimento de um incêndio. Foram considerados dois modos de transferência de calor (radiação e convecção) para a simulação. No caso de um confinamento total do compartimento, o fluxo de calor convectivo incidente na superfície frontal do bombeiro e nas costas do bombeiro permanece relativamente baixo $\left(<2,2 \mathrm{~kW} / \mathrm{m}^{2}\right)$ e tende a desaparecer devido às condições de ventilação. A superfície frontal é, no entanto, exposta a um fluxo de calor de radiação elevado $\left(\sim 6,8 \mathrm{~kW} / \mathrm{m}^{2}\right)$ que, apesar da diminuição da intensidade da chama, diminui para um valor limite $\left(0,5 \mathrm{~kW} / \mathrm{m}^{2}\right)$, que corresponde à potência de emissão do fumo em seu redor. Embora a ventilação mecânica atenue a intensidade do fluxo incidente e, consequentemente, a temperatura ambiente, ela pode contribuir para gerar uma ignição secundária na fonte do material combustível, induzindo, portanto, o fenómeno de incêndio generalizado (FOV).

\section{AGRADECIMENTOS}

O presente trabalho é financiado pelo organismo de pesquisa Argelino DGRSDT, sob o projeto № A11N01UN020120150001. 


\section{REFERÊNCIAS}

BABRAUSKAS, V.; PEACOCK, R. D.; RENEKE, P. A. Defining flashover for fire hazard calculations: Part II. Fire Safety Journal, vol. 38, no. 7, p. 613-622, 2003.

BACZKIEWICZ J.; PAJUNEN S.; HEINISUO M. Effect of radiation inside square hollow section under moderate non-symmetric fire. Fire Research, vol.2, no. 1, p. 25-30, 2018.

CHEN, X.; LU, S.; LIEW, K. M. An investigation of horizontal opening effect on pool fire behaviour in a confined compartment: A study based on global equivalence ratio. Journal of Fire Sciences, vol. 34, no. 1, p. 13-29, 2016.

CORRÊA, C.; Braga, G. C.; MENEZES, R. R. F.; SILVA, J. J. R.; TABACZENSKI, R.; JUNIOR, J. D. C. B.; OLIVEIRA, T. A. P. Natural fire tests in a dormitory with fire protected furniture. Fire Research, vol. 2, no. 47, p. 7-11, 2018.

DAHAMNI, S. Heat exchanges analysis of a firefighter garment, 1st National Conference on CFD \&Tech, Draria, Algeria, p. 23-24, 2016.

DESANGHERE, S. External structure heating determination due to fire, Phd Thesis, National Institute of Applied Science of Rouen, France, 301 p. 2006.

DOUADI, $\mathrm{H}$. Incoming fluxes prediction on a firefighter body, Master's thesis, Hassiba Benbouali University of Chlef, Algeria, 75 p, 2018. 
FU, M.; YUAN, M.Q.; WENG, W. G. Modeling of heat and moisture transfer within firefighter protective clothing with the moisture absorption of thermal radiation. International Journal of Thermal Sciences, vol. 96, p. 201210. 2015.

HE, J.; WANG M.; LI J. Determination of the thermal protective performance of clothing during bench-scale fire test and flame engulfment test: Evidence from a new index. Journal of Fire Sciences, vol. 33, no. 3, p. 218-231, 2015.

LASSUS, J.; COURTY, L.; et al. Estimation of species concentrations during a fire in a reduced-scale room. Journal of Fire Sciences, vol. 34, no. 1, p. 30-50, 2016.

MVOGO, P. O.; MOUANGUE R. et al. Building Fire: Experimental and numerical studies on behaviour of flows at opening. Journal of Combustion, p. 1-11, 2019.

NAYAK, R.; HOUSHYAR, S.; PADHYE, R. Recent trends and future scope in the protection and comfort of fire-fighters' personal protective clothing. Fire Science Reviews, vol. 3, p. 1-19, 2014.

ROSSI J, L.; SIMEONI, A. et al. An analytical model based on radiative heating for the determination of safety distances for wildland fires. Fire Safety Journal, vol. 46, no. 8, p. 520-527, 2011.

ZHANG, G.; ZHU, G.; YUAN, G.; HUANG, L. Methods for prediction of temperature distribution in flashover caused by backdraft fire. Mathematical Problems in Engineering, vol. 2014, p. 1-6. 